\title{
I'HOMME L'Homme
}

LHOMME Revue française d'anthropologie

171-172 | 2004

Musique et anthropologie

\section{Musique, identité et "ville-monde"}

Perspectives critiques

Martin Stokes

\section{(2) OpenEdition \\ Journals}

Édition électronique

URL : http://journals.openedition.org/lhomme/24951

DOI : 10.4000//homme.24951

ISSN : 1953-8103

Éditeur

Éditions de l'EHESS

\section{Édition imprimée}

Date de publication : 1 décembre 2004

Pagination : 371-388

ISSN : 0439-4216

Référence électronique

Martin Stokes, « Musique, identité et "ville-monde" », L'Homme [En ligne], 171-172 | 2004, mis en ligne le 01 janvier 2006, consulté le 30 avril 2019. URL : http://journals.openedition.org//homme/24951 ; DOI :

10.4000//homme.24951 


\title{
Musique, identité et "ville-monde"
}

Perspectives critiques

\author{
Martin Stokes
}

S LE TERME D’ «IDENTITÉ » était très prisé dans les années 1990, il est aujourd'hui source de nombreuses critiques, et ceux qui l'utilisent encore paraissent naifs ou dépassés. Cet article vise simplement à insister sur le fait que cette notion a encore fort à faire, dans un contexte de mondialisation qui, sans la modifier fondamentalement, la complexifie. Avant de développer cela, il est utile de rappeler les différents courants théoriques.

Le point de vue prédominant dans les sciences humaines et sociales, entre le milieu des années 1970 et celui des années 1990 (lorsque les critiques devinrent virulentes), se résume à l'idée que l'on produit de l'identité en entrant dans des rapports symboliques avec l'autre (principalement par le langage, mais non exclusivement) ; l'identité n'existe pas et ne peut exister avant ou indépendamment de ce processus. Les rapports impliqués sont complexes et souvent farouchement contestés ; certaines voix se font entendre, mais d'autres sont étouffées ; certains y sont extrêmement sensibles, d'autres sont à peine conscients de la nature de la situation dans laquelle ils sont tous impliqués. La psychanalyse, la sociologie et l'anthropologie ont éclairé une telle complexité, chacune à leur façon. Naturellement, la psychanalyse insista sur la dynamique affective de tels rapports, la sociologie sur ceux de pouvoir et l'anthropologie sur les processus de médiation symbolique, même si chaque domaine de recherche allait puiser de plus en plus et de manière fructueuse aux découvertes des autres. Toutes les disciplines soulignaient la nature incertaine de l'identification en tant que processus social qui produit l'«identité». Le point de vue scientifique de l'époque, qui considérait l'identité comme relationnelle, contextuelle et discursive, était en nette divergence par rapport au point de vue profane, pour qui l'identité est une conscience de soi, stable et prédéfinie, c'est-à-dire une intériorité subjective, qui exige une extériorisation dans une forme censée être "vraie " ou " authentique ".

Cependant, vers la fin du XXe siècle, les modes de pensée scientifique et populaire convergèrent dans la façon de concevoir l'identité, tout se compliquant. Les 
débats nés autour de la musique ont joué un rôle important. Paul Gilroy (1993), Les Back (1995), George Lipsitz (1994) et d'autres placèrent la musique au centre de l'expérience diasporique des esclaves africains dans le Nouveau Monde et ailleurs. Selon eux, la musique fournissait la matière dans laquelle l'ego (dialogique ou "antiphonal») se forgeait, non seulement en impliquant les autres mais en reconnaissant sérieusement et de façon positive leur présence à titre de "co-auteurs» de ces ego postmodernes. Cette notion fut couramment utilisée par une grande partie de la littérature consacrée à la musique du monde ou au phénomène du world beat vers la fin des années 1980 (Broughton et al. 1994). Les langages universitaires et populaires utilisés pour parler de l'identité se trouvèrent mêlés de manière complexe et féconde. Mais lorsque la façon d'envisager l'identité se mua en un tourbillon agrégeant une multitude de particules, il fut difficile de ne pas en arriver à la conclusion qu'il n'y avait plus, dès lors, de perspectives critiques.

Cela suscita une vive réaction qui, je crois, marque encore les débats actuels tournant autour de l'identité (et autour de sujets qui y sont liés tels que, par exemple, la race et l'ethnicité). Les spécialistes en musique populaire ainsi que les critiques littéraires noirs ont observé que la construction idéologique d'identités postmodernes prêchait une politique d'émancipation radicale, mais ils associaient de manière résolument obsolète la pensée critique à la culture avec un grand C, c'est-à-dire celle des textes et des interprétations (Frith 2000). Pour prendre un exemple éculé, l'album Graceland de Paul Simon avait été perçu, de manière beaucoup trop désinvolte et dépourvue de sens critique, comme un symbole du dialogue interculturel (Mentjes 1990 ; Erlmann 1996 et 1999). On finit par reconnaitre que les pratiques sociales réelles, à l'intérieur desquelles de tels textes se constituent et circulent, s'avéraient être beaucoup plus complexes et qu'elles pourraient être perçues comme renforçant justement ce qu'elles prétendaient subvertir (la division racialisée du travail dans le cas de Graceland de Paul Simon, par exemple). Il est difficile de ne pas en conclure que les vraies conditions de la production de la musique mondiale demeurent extrêmement mal comprises et sont complètement mystifiées par le discours que prêche l'industrie de la "world music» qui insiste sur des notions de dialogue et d'échange auxquelles d'ailleurs les chercheurs et spécialistes ont souscrit.

La théorie postcoloniale a pesé elle aussi sur le discours de l'identité dans les domaines plus conventionnels de l'analyse musicologique et ethnomusicologique. La critique acérée de Kofi Agawu (1992) à propos de l'ethnomusicologie africaniste, par exemple, décrit les précédentes tentatives pour définir le rythme africain, d'une part, comme étant motivées par une logique coloniale, et d'autre part, comme masquant sérieusement les processus rythmiques et métriques réellement mis en œuvre en les comparant toujours à ceux de la musique classique occidentale et en insistant sur leurs différences. Les questions que soulève Kofi Agawu sont les suivantes: pourquoi ne pas traiter la musique africaine simplement pour et en elle-même, et pourquoi les ethnomusicologues perpétuent-ils un apartheid méthodologique? Il est grand temps, d'après Kofi Agawu, que l'on 
commence à traiter les «autres musiques " en tant que musiques plutôt qu'en tant qu' « autres».

La «mondialisation» a finalement compliqué les débats concernant l'identité. Ce terme de mondialisation (ou de globalisation) a remplacé les notions, plus anciennes, de «modernisation » et d' «impérialisme culturel ", mais il perpétue les inquiétudes relatives à l'érosion des pratiques et des identités locales liées à ces deux notions. Les acteurs de la "mondialisation", telles les entreprises multinationales, les structures politiques régionales et transnationales, la main-d'œuvre et les marchandises qui circulent de façon omniprésente, sont perçus comme des dispositifs évoluant dans un espace lointain, abstrait, général et impersonnel, qui agissent sur la pratique locale. Quel que soit cet espace, on ne peut utiliser le langage habituellement associé à la localité, qui, pour en parler, implique les concepts d'ethnicité, d'identité, de race, etc. On envisage de plus en plus la mondialisation en termes de nouvelles formes de civilité et de cosmopolitisme ; les identités locales qu'elle définit ont tendance à être comprises soit comme des réactions primaires, soit comme faisant partie du processus capitaliste qui consiste à lancer sur le marché des produits fondamentalement semblables tout en faisant valoir leur différence.

On se trouve ainsi face à deux problèmes que cet article entend faire ressortir. Le premier est le danger de jeter le bébé avec l'eau du bain. Les catégories identitaires auraient été forgées dans le contexte biaisé et violent de la rencontre coloniale et continueraient ainsi à déformer la façon dont nous saisissons et mettons en relation les éléments inclus dans ces catégories, et ce quel que soit le côté du fossé postcolonial où l'on se trouve. Elles font néanmoins partie des mondes musicaux dans lesquels nous continuons de vivre ; elles ont des dimensions institutionnelles et idéologiques; elles continuent à conditionner le langage et donc notre façon de penser, de parler et d'agir dans le domaine de la musique. Mais il y a certainement d'autres motifs de préoccupation. D'une part, on ne peut entièrement réduire l'activité musicale à la production d'une identité et, d'autre part, il existe bien d'autres manières de produire de l'identité. Mais on ne peut pas non plus ignorer l'existence de catégories identitaires dans l'activité musicale.

Deuxièmement, la tendance à considérer «le mondial » comme une abstraction sans localité (ainsi que «le moderne » avant lui), finit par le placer au-delà de toute considération historique et donc critique; par conséquent, les réactions « locales " paraissent souvent irrationnelles, anarchiques, voire vaines sur un plan politique. Ce texte tente de déterminer les circonstances particulières d'un cas de construction d'identité à travers la musique. La "formation identitaire " apparâ̂trait ainsi, non pas comme une microréaction à un macropouvoir, mais comme un processus ordonné et systématique qui articule et structure les formes spécifiques d'action au sein de la culture d'entreprise, de la diaspora des travailleurs immigrés, de la ville et de l'État-nation. Ce qui suit examinera donc ce processus à la lumière de l'exemple particulier de l'appropriation du rap et du hip-hop par des communautés d'immigrés dans des villes d'Europe. 


\section{"Musique noire" et "Vieux Monde"}

374 Le degré d'antipathie envers la culture populaire américaine est très élevé dans l'intelligentsia européenne, alors que pour beaucoup d'immigrés et de marginaux des villes européennes comme Londres, Berlin et Paris, le lien entre leur expérience et celle du ghetto afro-américain demeure puissant et indéniable (Back 1995-6, Sharma et al. 1996, Robins et Morley 1996, Stokes 2002, Gross 1997, Virolle 1995). Les jeunes de la génération élevée au son de musiques visant un public immigré telles que le bhangra, le raï et l'arabesk des années 1980, se sont tournés vers le rap et le hip-hop dans les années 1990. Ils y ont trouvé une marque de distinction générationnelle et une façon de répondre à la vague montante d'hostilité néonazie envers les «Noirs» d'Europe. Le processus de «transfert » d'identité a cependant été complexe et tumultueux.

Cartel, un groupe de rappeurs turco-allemands, apparut à Istanbul en $1995 \mathrm{au}$ milieu d'une véritable tempête médiatique. Pour ses supporters, que l'on comptait parmi les membres de l'intelligentsia, ce jeune mouvement culturel allait enfin conquérir le monde. Ceux qui furent autrefois la cause d'une gêne, les gastarbeiter turcs (travailleurs immigrés en Allemagne), devinrent ainsi, du jour au lendemain, une ressource valorisée et valorisante. Certains spécialistes turcs (Bora 1996) avaient aussitôt démontré que ce qui était présenté comme une grande nouveauté était simplement le dernier stade dans l'histoire d'un siècle d'appropriations délibérées de la culture musicale populaire occidentale par les Turcs. On pourrait d'ailleurs en tracer, ajoutèrent-ils, une ligne droite qui irait de l'appropriation turque de l'opérette, sans compter le tango, la chanson, le rock et la pop, au rap et au hip-hop. Au fil des siècles, les Turcs s'étaient emparés avec succès de nombreux biens culturels étrangers. Cela n'était pas une critique. Des intellectuels turcs estiment que la capacité d'absorption et d'appropriation de leur société a façonné son identité culturelle. Les spécialistes d'études culturelles précisent, quant à eux, que ce qui, parfois, semble être à première vue une pure imitation, est bien souvent la résultante d'un processus complexe, dynamique et créatif. D'après eux, nous pourrions en apprendre davantage sur notre propre identité en écoutant attentivement ce que Cartel ainsi que d'autres TurcoAllemands ont à dire.

D'autres circonstances relancèrent le débat. Cartel avait également été chaleureusement accueilli par de jeunes Turcs fascistes dont l'idéologie, qui proposait un mélange peu attrayant de nationalisme racialisé et d'islam orthodoxe, avait été élaborée par Alparslan Türkeş dans les années 1960, et revenait à la surface à cause du climat politique libéral de la fin des années 1980. Leur indignation provoquée par la situation humiliante des Turcs en Allemagne avait été attisée par une série d'agressions néonazies, dont l'incendie criminel de Sollingen en 1995, où des gastarbeiter furent les principales victimes. Cela finit par déclencher (du côté de l'extrême-droite turque) des ripostes, et des bandes s'organisèrent, s'exhibant sous le drapeau turc et affichant un croissant islamique sur leurs habits. D'après eux, les dazlaks (skinheads néonazis) allaient avoir ce qu'ils méri- 
taient depuis longtemps! La presse libérale en Turquie réagit avec inquiétude. Lintelligentsia, autrefois enthousiaste, fit marche arrière. Attentive aux susceptibilités de la clientèle bourgeoise qui achetait ses CD, la maison de disques RaksPolygram de Cartel se fit discrète. Des concerts furent annulés. Leur album devint de plus en plus difficile à trouver. À peu près neuf mois après sa sortie, $j$ 'ai eu moi-même du mal à le trouver, mais je finis par en dénicher un exemplaire dans l'un des points de vente de Raks en banlieue. Je me souviens qu'il mavait fallu le chercher dans une boîte à chaussures sous le comptoir. Les seuls endroits où je l'avais vu bien mis en évidence, c'était chez des vendeurs de cassettes itinérants, opérant aux principaux terminus des transports en commun vers les banlieues éloignées. Le marché des cassettes et celui des CD formaient alors deux mondes à part, et Cartel semblait avoir pu se glisser entre les deux.

Il est étonnant que les débats autour de Cartel se soient déroulés sans faire référence ou presque à leur musique même, ou au contexte musical d'où elle était issue. Pour ne pas engager la discussion dans des considérations trop générales, quelques précisions descriptives et comparatives (nécessairement limitées) s'imposent. La scène du rap turco-allemand d'où venait Cartel était très active dans les années 1990. À quelques exceptions près (notamment la rappeuse féministe Aziza A), les rappeurs turcs et les artistes hip-hop en Allemagne ont été fiers de se positionner eux-mêmes en marge de la création musicale populaire allemande ; ils se sont confinés aux meetings antiracistes, aux clubs de Kreuzberg (dans le centre de Berlin) et autres lieux réservés à la création musicale «immigrée ", aux studios d'enregistrement dans lesquels l'avant-garde de la musique populaire crée de nouvelles alliances interculturelles, et aux sites internet (notamment les forums de discussions où s'échangent des fichiers MP3), que l'on peut, d'ailleurs, considérer comme l'institution qui est au cour de la culture rap et hip-hop d'aujourd'hui.

Erci E, Karakan et Da Crime Posse ("Cinai Sebeke») s'étaient réunis en 1993 pour enregistrer un disque sous le nom de Cartel. La sortie de ce CD coïncida avec celle de l'album d'un autre groupe basé à Francfort composé de DJ Mahmut, DJ Volkan et KMR. Il peut être utile de comparer ces deux albums, en s'intéressant non pas au rap de Berlin et au hip-hop de Francfort, mais aux différentes stratégies adoptées par les rappeurs. Ces stratégies consistent à choisir une orientation soit vers la diaspora turque, soit vers la culture musicale underground allemande. Alors que l'enregistrement de Cartel ciblait les Turcs de Turquie et la grande communauté d'immigrés en Allemagne, le groupe basé à Francfort s'adressait manifestement aux rappeurs et artistes hip-hop d'avantgarde en Allemagne.

Cette distinction se ressent très nettement dans la façon d'utiliser le sampling*. Dans la musique hip-hop, le sampling est souvent compris comme exprimant une sorte de détachement, d'ironie postmoderne; mais, dans le cas de

* De «sample» (verbe et substantif signifiant échantillonner et échantillon), le sampling désigne l'opération d'échantillonnage qui consiste, grâce aux techniques de l'enregistrement numérique, à prélever et recycler des fragments sonores de morceaux déjà enregistrés suivant des fréquences doublées par rapport au signal échantillonné afin d'éviter les distorsions (i. e. $44,1 \mathrm{kHz}$ ou $48 \mathrm{kHz}$ ). $N d l$ r. 
Cartel, le sampling renvoyait à la fierté des rappeurs turcs vis-à-vis de leurs racines, coutumes et traditions. Les sons turcs prédominaient clairement: des sons associés à l'instrumentation rurale (c'est-à-dire des sons " turcs » d'après des critères folkloriques bien établis et promus par l'État turc) : le $s a z$ (un luth à manche long), le zurna et le davul (la bombarde et le tambour). Les samplers préservent l'identité des phrases musicales dans lesquelles ils sont prélevés, avec très peu de distorsion et d'interruption. La première plage du CD (Cartel, Bir Numera...) introduit un extrait de chœur de femmes, prélevé de façon évidente selon certains auditeurs de ma connaissance, dans la célèbre émission de musique folklorique Yurttan Sesler (Les voix de la patrie). Cet extrait est très caractéristique tant sur le plan mélodique que lexical et on ne peut se tromper quant à l'origine " authentique » de la tradition turque. Le tempo des rythmes de base du rapping correspond à celui de la çifte tell, danse (de couple) turque. Musicalement, le sampling joue sur les attentes d'un public familiarisé avec la musique turque diffusée largement par les radios, et capable d'identifier des sons spécifiques comme " authentiquement " turcs.

L'utilisation du sampling sur le CD de DJ Mahmut est tout à fait différente : il peut s'agir aussi bien de boucles de jazz et de piano-bar, que de sons industriels ou de machines provenant d'une instrumentation souvent utilisée dans le monde de la création musicale expérimentale allemande. Les références musicales turques sont rares; elles sont prélevées dans la tradition de la musique savante urbaine (des fragments d'improvisations taksim et des refrains instrumentaux) et non dans la tradition "turque" rurale. Lorsque des sons "turcs" se font entendre, comme par exemple lors d'un passage taksim improvisé à la clarinette qui fonctionne comme un court break instrumental de Bastir, ils sont plus fragmentés et jouent sur des variations rythmiques. Autrement dit, ils sont intégrés dans leur totalité, mais sont méconnaissables, tel un matériel acoustique brut destiné à être transformé par les musiciens, plutôt qu’à marquer l'authenticité comme chez Cartel.

Par ailleurs, la distinction entre les deux groupes se repère également dans la langue utilisée. Les membres de Cartel ne rappaient pas uniquement en turc mais en anglais, en allemand et en espagnol, soulignant ainsi, avec une fierté évidente, les capacités d'adaptation à différents codes linguistiques ou autres, que les immigrés turcs ont acquis dans des villes européennes. De nombreux articles sur Cartel ont mis en évidence la politique ethnique utopique sous-jacente au multiculturalisme de leur langage parlé et rappé. Mais la plupart des textes sont écrits dans un turc assez facile à comprendre et traitent de façon claire d'un immigré turc non métissé qui savoure le fait d'être turc en vivant toutes sortes de vengeances fantasmées et de rencontres sexuelles avec des Allemandes dans les rues, dans les clubs et dans les bars de Berlin. L'auditeur-cible est de toute évidence un jeune Turc de métropole en accord avec le style sexiste tapageur de l'Istanbul laxiste du début des années 1990, emprunté à une culture publique urbaine de "delikanlllik», c'est-à-dire une attitude de défi machiste. Les paroles scandées par DJ Mahmut et al. sont assez différentes: elles sont composées d'un mélange à 
parts égales de turc et d'allemand, et la langue peut changer à l'intérieur d'une phrase ou même d'une proposition. Elles traitent de problèmes raciaux sur fond d'aliénation urbaine et d'angoisse où domine une atmosphère de gravité méditative, parfois désespérée. La plupart des Turcs nés en Turquie que je connais n'arrivaient pas du tout à comprendre les paroles. Il y avait une composante d'allemand assez importante pour qu'un locuteur allemand, sans avoir aucune notion de turc, puisse suivre l'essentiel des titres du CD. Les paroles de DJ Mahmut parlent d'une sous-culture immigrée en Allemagne et s'adressent à cette sous-culture et non à une diaspora turque plus large.

Les promoteurs de Cartel avaient tenté de conquérir le marché populaire en Turquie et celui de la diaspora turque, au sens plus large du terme. Cela plaça Cartel en marge de la culture underground allemande dont DJ Mahmut, Volkan, KMR, et d'autres après eux, furent plus représentatifs. Mais leurs efforts n'aboutirent pas. Malgré l'importance de Raks, leur distributeur (sur lequel je reviendrai plus loin), l'industrie du disque turque y est restée indifférente. Le système médiatique géré par l'État en Turquie était réfractaire à tout sauf à la pop et à la musique classique occidentale, et aux musiques savantes et folkloriques turques. Au milieu des années 1990, le rap restait en marge des «bars à rock » d'Istanbul, qui étaient en fait des clubs de centre ville pour étudiants, très peu chers, et qui visaient davantage à perpétuer la longue tradition de rock turc qu’à créer un espace ouvert à de nouvelles aventures esthétiques de ce genre. Bien que l'on considère les membres de Cartel comme les grands-pères du rap et du hip-hop turcs, ils n'ont pas de descendants directs en Turquie dans le courant dominant de la musique populaire. De plus, le fossé entre Berlin et Istanbul que Cartel avait promis de réduire au nom d'une jeune culture libératrice demeure toujours aussi large.

\section{World music et culture d'entreprise}

Plusieurs facteurs peuvent être envisagés pour rendre compte de l'échec de la tentative de Cartel de vouloir créer une nouvelle culture turque pour les jeunes de la diaspora, lequel échec fait apparaître les limites d'une politique identitaire romantique face à la puissance des systèmes modernes abstraits, à savoir les entreprises multinationales, les villes et les dispositifs médiatiques en place. Mais il peut aussi suggérer d'autres limites, celles de la conception que les musiciens ont de leur propre identité dans le contexte formé par de telles puissances. Les débats autour de Cartel, auxquels plusieurs critiques et intellectuels ont pris part, tendent vers l'une ou l'autre de ces directions. Le tableau serait quelque peu différent et forcément plus complexe si l'on considérait que les forces paradigmatiques de ce qu'on pourrait appeler la «modernité mondiale abstraite " participent de façon non négligeable à la construction d'identités. Trois sites de la «modernité mondiale abstraite » sont à prendre en considération dans cette discussion et, en fait, peuvent être vus comme les sites abstraits les plus représentatifs de cette mondialisation : l'industrie phonographique, la ville et l'État-nation. C'est donc à la fois de 
façon générale et en fonction de notre exemple précis que je vais examiner chacun de ces éléments.

L'industrie phonographique représente un point de départ important et tout à fait indiqué puisque beaucoup d'ethnomusicologues et autres théoriciens de la culture l'ont associée au phénomène d'expansion de la "grisaille mondiale ", voire à l'érosion des différences musicales locales et des identités. Les sociétés multinationales et plus particulièrement Time-Warner, Thorn-EMI, Bertelsmann, Sony, PolyGram, Matsushita - qu'on appellera dorénavant les majors - ont exercé un contrôle total sur la plus grande partie du paysage sonore au sein des pays industrialisés. En dehors de ces marchés "développés », les majors ont déployé des efforts considérables pour endiguer sinon éradiquer le piratage des cassettes, des ondes et, à présent, les fichiers musicaux numérisés à travers les sites internet; ils ont écarté du marché leurs rivaux, particulièrement les producteurs locaux de cassettes, pour contrôler là aussi ce que le public écoute. Le cas turc est typique à beaucoup d'égards. La diffusion de la musique par les cassettes et la radio a été essentiellement une affaire nationale jusqu'à la fin des années 1980, étendue bien sûr aux gastarbeiter turcs et kurdes en Europe. La libération politique et économique de la fin des années 1980 a introduit les CD, la radio FM et les chaines d'émission musicale par satellite. Dès lors, la production et la consommation musicales ont commencé à prendre une dimension mondiale, les $\mathrm{CD}$ étant dorénavant fabriqués avec des technologies de pointe dans divers endroits du monde. Un public « mondial » est devenu l'ultime arbitre du succès et de la valeur culturelle.

Au niveau de l'industrie phonographique turque, l'essentiel de ce discours de la mondialisation eut une finalité locale, en déclenchant notamment des dynamiques particulièrement actives. C'est ainsi qu'une société de production saisit l'occasion et réagit sans tarder: le label Raks. Avant le milieu des années 1990, Raks avait signé avec presque toutes les principales vedettes de la pop et du rock, établissant ainsi un niveau de production très élevé, sous l'égide donc des valeurs de la mondialisation, et l'entreprise se lança dans des campagnes de publicité fort coûteuses. La pression qu'elle exerça pour l'harmonisation d'une loi de droits d'auteurs européenne afin de lutter contre le piratage des cassettes eut moins de succès, mais elle fit beaucoup de bruit. Tous ces efforts portèrent rapidement leurs fruits et un accord fut signé avec le label PolyGram. Le premier acquis de cet accord fut que, pour la Turquie, Raks réussit à obtenir les droits de distribution de Mercury, la filiale allemande qui s'était chargée de la production et de la diffusion de Cartel.

Le cas de Cartel illustre donc l'histoire désormais bien connue de l'engagement des majors du disque sur les marchés de musique du Tiers-Monde encore peu développés, avec leur politique d'absorption d'industries locales, la propagation, par leurs soins, de styles musicaux occidentaux et l'effacement des identités régionales. On pourrait aisément interpréter ce processus en termes d' «impérialisme culturel »; mais cette expression large occulte les relations spécifiques et idiosyncrasiques plus localisées, qui existent entre les forces commerciales du "centre " et celles de la "périphérie ». Car les majors sont implantées dans des lieux parti- 
culiers et ont des cultures commerciales et des façons de concevoir l'identité d'entreprise qui dépendent fortement des circuits dans lesquels les sons mondiaux circulent. Thorn-EMI, par exemple, avait hérité des titres de Columbia et de Gramophone en 1931, deux des sociétés ayant promu et exploité des marchés importants de musique locales du monde colonial du début du XXe siècle (Burnett 1996). Thorn-EMI était la seule parmi les majors à mettre à disposition des ressources et à déployer de considérables efforts pour investir et exploiter l'immense marché des musiques au Moyen-Orient.

Pour revenir à notre cas, Raks s'était liée avec PolyGram afin de promouvoir Cartel pour des raisons précises et locales à côté desquelles on risquerait de passer si on se limitait à ne voir dans PolyGram rien d'autre qu'une puissance " mondiale », lointaine et abstraite, qui n’opérerait qu’à distance. PolyGram, qui fut le produit, en 1972, d'une fusion entre Phonogram, société néerlandaise, et Polydor, société allemande, avait lancé immédiatement une campagne agressive pour acquérir de nombreuses maisons de disque indépendantes à travers l'Europe, dont Barclay, une société française (laquelle Barclay connut un best-seller avec la vente, tant aux amateurs européens de world music qu'au public nordafricain résidant en France et au Moyen Orient, de l'album Didi que le chanteur de raï algérien Khaled avait réalisé en 1992). L'expérience de ces marchés complexes et la présence de grandes communautés de gastarbeiter turcs en Allemagne et aux Pays-Bas devaient rendre l'idée du partenariat avec Raks et de la promotion de Cartel attrayante. Cela suggère, entre autres, que les anciens colonisateurs continuent à exercer un contrôle économique et culturel important sur leurs colonies, anciennes et nouvelles, ce qui semblerait confirmer l'hypothèse de l'impérialisme culturel, au moins à un certain niveau. Cela suggère aussi qu'un tel contrôle dépend du contexte culturel et historique. Mais le contrôle des marchés locaux par les entreprises «mondiales" n'est ni abstrait, ni total, ni inévitable comme l'hypothèse de l'impérialisme culturel le laisserait entendre. Il est, au contraire, rigoureusement déterminé par le lieu, l'histoire et l'identité.

\section{Culture migratoire et identités à trait d'union}

Les débats autour de la mondialisation sont très nettement axés sur la circulation des marchandises et des capitaux. Pour de nombreux partisans de la mondialisation, les systèmes abstraits impliqués dans la circulation des marchandises et des capitaux ont un rôle civilisateur à jouer qui dépasse les spécificités locales. Les mouvements de migration, eux, sont ignorés ou traités avec ambivalence dans ce contexte de mondialisation (Sassen 1998). Il n'est pas difficile d'en imaginer les raisons. Les immigrés apportent avec eux des formes culturelles qui sont perçues par ceux qui assignent une mission civilisatrice à la libre circulation des marchandises, à la division mondiale du travail, à l'urbanisation généralisée, etc., comme tenaces, intrusives et perturbatrices.

Les mouvements mondiaux de migrants des classes très défavorisées dans les villes d'Amérique du Nord et d'Europe de l'Ouest sont aussi vitaux que la circula- 
tion des marchandises pour la création et la transmission des cultures «mondiales ». Cela s'applique aussi bien au domaine de la musique. Les analyses ont de plus en plus porté leur attention sur les dimensions mondiales de la culture migratoire dans les villes des pays industrialisés au cours des deux dernières décennies. L'essor de ce qu'on pourrait appeler les «identités à trait d'union " (turco-allemandes, anglo-asiatiques, etc.) a été salué comme une force capable d'ébranler l'instrument oppressif de la production d'identité par l'État-nation, ne serait-ce que par la mise en jeu de nouvelles notions d'appartenance, infiniment diversifiées et ouvertes. C'est ainsi que le rôle joué par les formes musicales afroaméricaines dans le défi posé aux identités locales hégémoniques et dans l'établissement de nouvelles alliances culturelles entre les marginalisés et les dépossédés dans les sociétés cloisonnées a fait couler beaucoup d'encre (Robins \& Morley 1996, Lipsitz 1994, Sharma et al. 1996). On ne peut plus croire aujourd'hui que la mondialisation soit un facteur d'homogénéisation culturelle ou celui d'un statu quo politique, comme les partisans de l'« impérialisme culturel» tentaient autrefois de nous en convaincre.

Bien qu'elle pût plaider en leur faveur, l'idée que Cartel aurait forgé une identité «turco-allemande» soulève de nombreuses questions. Une telle affirmation contribue à marginaliser les Turcs dans la société allemande En effet, elle limite la composante "turque" de l'identité turco-allemande à une catégorie unitaire où les éléments "moyen orientaux " et «islamiques» sont indifférenciés. Cela élude la question de l'appartenance à l'une ou l'autre de ces deux catégories, distinction pourtant essentielle dans la vie diasporique. Presque tous les Turcs d'Allemagne sont musulmans, mais ils ne se définissent pas tous en termes de confession religieuse. Ceux qui sont nés en Turquie ont été élevés dans un État profondément lä̈que. Ceux qui se rapprochent des organisations islamistes transnationales et qui s'affichent ouvertement en Allemagne, comme l'organisation Rabite des années 1980, n’ont aucun lien avec l'islam noir américain qui anime et domine le monde du rap.

Cette affirmation soulève également des questions d'ethnicité. En Allemagne, les Kurdes et les Turcs vivent souvent dans les mêmes endroits et ils se sont souvent associés, que ce soit pour lutter contre le néonazisme ou pour défendre leurs intérêts communs de travailleurs immigrés dans ce pays. Mais ils ont connu aussi des frictions et des moments de rupture : la guerre entre l'armée turque et le PKK séparatiste kurde dans les années 1990 créa des divisions importantes dans les quartiers d'immigrés en Allemagne et en France (Kastoriano 1995). La tactique de Cartel qui consista à réunir les symboles du nationalisme turc avec l'une des expressions musicales de l'islam afro-américain a été mal interprétée par ceux qui voyaient dans le lien tressé entre l'islam et la Turquie, que ce soit en Allemagne ou en Turquie, quelque chose d'essentiel plutôt qu'une position stratégique et provisoire.

En fait, c'est une erreur de penser les «identités à trait d'union" issues de migrations comme étant systématiquement en opposition ou occupant un espace sous-culturel vierge. La popularité de Cartel coïncida avec un engouement pour 
"le chic à la turque " (migrant chic) qui se répandit en Allemagne au milieu des années 1990, lequel fut cultivé dans Der Spiegel, Die Zeit-Punkte et autres journaux sélects par toute une série d'articles sur la mode turco-allemande, le cinéma, les bijoux, la nourriture, les boissons et l'attraction que représentaient les quartiers d'immigrés (certes observés à distance). Les identités "à trait d'union " devinrent à la mode, véhiculant ainsi une image d'émancipation et de cosmopolitisme. Bien sûr, tout cela ne fut pas forcément négatif. Mais le «chic à la turque " entretenu par les intellectuels empêcha de se confronter aux racines de la discrimination envers les Turcs dans la société allemande et, en fin de compte, ne fit qu'envenimer davantage le conflit avec des groupes néonazis issus de la classe ouvrière. Dès lors, en attaquant les Turcs, ceux-ci s'en prenait du même coup à l'élite libérale, laquelle était pour eux tout aussi haïssable. En outre, l'idéalisation de la vie des immigrés prit une valeur normative et constitua un critère inflexible par rapport auquel fut jugée la civilité des immigrés. En ce sens, les identités "à trait d'union" sont tout aussi déterminantes que les identités "simples" auxquelles elles sont constamment opposées. En cette unique catégorie de "turco-allemand", étaient mis dans le même sac des groupes de gens différents ayant très peu de choses en commun et dont les intérêts n'étaient pas nécessairement servis à la lumière de cette homogénéisation forcée.

Le langage introduit une inclusion complaisante qui dissimule de même les enjeux relatifs au genre et à la sexualité. La posture machiste de Cartel peut certainement être interprétée, et dans une certaine mesure excusée, par les liens musicaux stratégiques qu'ils essaient d'établir entre l'expérience des immigrés noirs américains et celle des immigrés européens. Mais cette posture machiste révèle aussi autre chose : elle exprime les inquiétudes liées au genre et à la sexualité qui accompagnent le processus de mondialisation. Alors que le monde célébrait la chute du mur de Berlin, les Turcs furent tiraillés dans toutes les directions. La main-d'œuvre est-allemande à bon marché remplaça rapidement la main-d'œuvre turque. La chute du mur de Berlin transforma les quartiers immigrés autrefois marginaux de Berlin, celui de Kreuzberg notamment, en parcs immobiliers aux loyers exorbitants, lesquels loyers furent l'objet de toutes sortes de pression qu'exercent les entreprises dans toutes les "villes mondiales " sur ceux du centre ville. Dans un climat économique général de réduction de personnel, d'embauche d'intérimaires et de délocalisation, les industries de services privilégièrent la main-d'œuvre féminine. Dans de nombreux foyers de gastarbeiter, les salaires des femmes devinrent la principale ressource des familles, et les hommes se sentirent de plus en plus déphasés au sein de l'économie domestique. Le ton sexualisé du discours d'émancipation de Cartel pourrait ainsi s'expliquer par une manière de faire face à une situation où les gens - et les hommes surtout - font l'expérience de processus de "déqualification " (dis-empowerment), sous de telles formes, directes et intenses, quelles ne peuvent que générer des tensions entre hommes et femmes. 


\section{Nation, État et "ville-monde"}

Les théories de la mondialisation voient souvent les États-nation et les identités qui leur sont associées comme étant en déclin. Au fur et à mesure que la mondialisation progresse, la capacité de l'État-nation à structurer des domaines tels que le politique, l'économique et le culturel s'amoindrit. Mais à s'en tenir à de telles considérations, on ne peut qu'escamoter le fait que ce sont les États-nation eux-mêmes qui ont favorisé la coopération avec des sociétés multinationales, en créant des zones industrielles, des zones d'affaires et de loisirs dans leurs plus grandes villes et ce afin de faciliter les besoins de ces entreprises (Sassen 1998). On peut reconnaître immédiatement les effets d'un tel processus à New York, Londres, Berlin, Bombay, Tokyo, Istanbul, Sao Paolo et ailleurs, qui affichent la fierté civique d'avoir atteint le statut de "ville-monde». On parla beaucoup du statut "mondial " d'Istanbul lorsque la ville accueillit le colloque " Habitat II " en 1996, à l'occasion duquel les autorités municipales avaient fait complètement nettoyer le quartier des affaires du centre ville, supprimant les taudis, fermant les bars et les bordels (dont le principal lieu de prostitution transsexuelle). Un vaste réseau de nouveaux ponts et de nouvelles artères relia des usines de plus en plus dispersées à des centres d'affaires, à la banlieue proche et à la grande banlieue. Ici, comme ailleurs, l'État s'est beaucoup impliqué dans la création d'un contexte où puisse s'installer la mondialisation, et il est loin d'en avoir été la victime passive. Cela nous conduit simultanément à traiter du troisième lieu et acteur - l'Étatnation - et à amorcer une conclusion.

L'État-nation continue certes à façonner l'expérience culturelle à travers le monde de manière directe et décisive, en tandem et non en opposition avec la mondialisation. Les systèmes nationaux de transmission des ondes (radio, télévision) jouent encore un rôle important dans la production de musique locale. Les États-nation protègent leurs propres industries culturelles des forces mises en œuvre dans la mondialisation par tout un système de quotas, lesquels demeurent toujours opérationnels dans un certain nombre d'États européens. La transmission par satellite et par internet a pu certes poser quelques problèmes, mais, ces dernières années, elle a plutôt incité ces États à adapter la législation en vigueur ou à en créer une nouvelle. Les effets des stratégies à la fois protectionnistes et expansionnistes des entreprises de médias peuvent cependant être complexes et imprévisibles comme l'illustre, à nouveau, le cas de Cartel. Au sommet de sa campagne contre le PKK, le gouvernement turc fut en mesure d'exercer une pression sur la BBC pour qu'elle cessât toute transmission par satellite des émissions de la chaîne turco-kurde, MED-TV, basée à Londres, lesquelles étaient reçues en Turquie. Ce satellite permettait également au public turc de recevoir MTV. Si l'on voulait arrêter la transmission de l'une des chaînes, il fallait forcément arrêter les deux. MTV ne s'est pas préoccupée de cela (je ne sais d'ailleurs pas pourquoi). À ce moment-là, cependant, un marché de programmes de musique dans le style de MTV venait d'émerger en Turquie. Kral, une société de diffusion par 
satellite turque, avait vite saisi cette occasion et occupé l'espace hertzien vacant en préparant et diffusant un programme dans le style de MTV mais avec un contenu musical turc. En 1996, Kral devint la principale vitrine pour des sociétés cotées comme Raks; un clip transmis en boucle était le prix à payer pour toute nouveauté. Une grande partie de la publicité faite autour de Cartel à Istanbul fut donc liée aux clips qu'ils réalisèrent et qui furent régulièrement transmis et repris sur Kral TV. De sorte que la circulation de la musique à travers les médias d'échelle pourtant mondiale doit encore beaucoup à l'intervention, volontaire et involontaire à la fois, de l'État-nation.

Si l'État joue donc un rôle important dans la production des identités à l'ère de la mondialisation, qu'en est-il de la nation ? Contrairement à ce qui est d'ordinaire avancé, je dirai que la circulation de formes culturelles hybrides tend à renforcer les sentiments d'appartenance nationale plutôt qu'à les diluer. Comme je l'ai déjà indiqué, les productions musicales ne sont pas à même de définir des réponses univoques de la part du public. Le discours d'émancipation turcoislamique de Cartel avait été en effet mal interprété. Pour les libéraux allemands, il véhiculait l'image d'un "chic à la turque ", mais aux yeux des conservateurs et de groupes racistes néonazis il venait confirmer le fait que la culture des gastarbeiter était profondément étrangère et, ce faisant, non assimilable. Ce même message de Cartel fut différemment interprété en Turquie. Les samples issus du folklore turc, et utilisés avec ironie, pouvaient être perçus comme une marque d'approbation du rôle de l'État dans la préservation de la culture nationale. Mais la juxtaposition d'images turques et islamiques ne pouvait être qu'explosive à un moment où, en raison de la récente élection d'un gouvernement islamiste, l'héritage entier de l'État laïque d'Ataturk risquait de vaciller. Le rude langage et la posture machiste de Cartel semblaient aussi s'adresser aux nouvelles classes engendrées par deux décennies d'adaptation aux préceptes du commerce multinational en Turquie. Ceux qui étaient exclus des retombées de ces nouvelles affaires avaient de quoi être dépités, et Cartel leur fit comprendre qu'ils avaient le pouvoir d'inquiéter les élites libérales qui se dépêchaient de promouvoir le nouveau statut "mondial » de la Turquie. Là, comme dans de nombreuses autres régions d'Europe, un nationalisme extrême s'est développé dans une atmosphère d'inquiétude vis-à-vis du phénomène de la mondialisation et de ses conséquences pour ceux qui sont économiquement défavorisés.

Il faut donc être très vigilant face aux diverses manières dont un " texte " donné traitant de politique d'identité, peut être interprété par différents groupes de personnes et tenir compte des conséquences sociales de tels actes d'interprétation et de la façon dont ils s'influencent les uns les autres. D'une part, le rap turc et le hip-hop parlent de formes d'identification mondiale qui transcendent l'État-nation. D'autre part, ils fournissent des éléments grâce auxquels une forme spécifique de "localité », l'État-nation, pourrait être identifiée et défendue avec acharnement en Turquie comme à Berlin. Ce n'est pas simplement que « ces deux interprétations soient justes chacune à sa façon ». C'est plutôt qu'elles émergent de positions individuelles différentes dans un espace partagé. Il n'y a 
certainement aucune raison de croire que la nation est en passe de perdre son pouvoir de structurer le sentiment le plus fondamental d'appartenance, bien que les gens puissent maintenant imaginer (à travers la musique et bien d'autres choses) différentes manières d'articuler le national à des catégories d'appartenance de niveau supérieur.

J'ai orienté mon propos vers une approche plus graduelle de la compréhension des identités, surtout en ce qui concerne leurs liens avec les pratiques musicales et avec les processus, les lieux et les modes de vie considérés aujourd'hui comme «mondiaux». Certains problèmes demeurent. Le tournant de ces dernières années dans l'analyse des discours sur l'identité a mis l'accent sur la circulation de certaines formes de marchandises, en particulier comme on l'a vu, les cassettes et les CD (faisant de la musique une notion réifiée, abstraite et quasi autonome), au détriment d'une attention portée sur les vies auxquelles elles sont mêlées. Cependant, cela n’a pas été complètement inutile. Le fait de percevoir des discours comme spécifiquement impliqués dans la production d'identités a permis d'aborder de façon plus nuancée les textes que l'on associait autrefois à la culture savante (qu'il s'agisse de formes littéraires, musicales ou autres), et de s'intéresser à leur implication sociale. Le défi, me semble-t-il, n'est pas tant de rejeter les " textes" en faveur des « contextes " et de s'enfermer par là dans une vieille opposition stérile, mais d'essayer de prendre les deux en considération, comme deux processus qui s'informent l'un l'autre. La musique concentre et condense les relations sociales (elle en est le produit); ces relations se présentent à nous sous formes objectivées et compressées et ont un impact sur notre corps et notre esprit. Ce que la musique nous apprend sur nous-même a un sens social et contribue pour une grande part à déterminer notre façon d'agir dans le monde, dans un rapport aux autres. Il faut certainement veiller à ne pas surestimer l'importance des textes dans la production des identités; mais on ne devrait pas non plus les ignorer.

Traduit de l'anglais (États-Unis) par Giancarlo Siciliano, revu par la Rédaction.

MOTS CLÉS/KEYWORDS : hip-hop - world music - identitélidentity - mondialisation/globalization - migration. 
Agawu, Kofi

1992 "Representing African Music»

Critical Inquiry 28 (1) : 245-66.

Appadurai, Arjun

1990 "Disjuncture and Difference in the

Global Economy ", in Mike Featherstone, ed., Global Culture : Nationalism,

Globalization and Modernity. Londres, Sage.

\section{Ardener, Edwin}

1989 "Belief and the problem of women ", in Malcolm Chapman, ed., The Voice of Prophecy. Oxford, Blackwell : 72-85.

Back, Les

1995 «X Amount of Sat Siri Akal. Apache Indian, Reggae Music and the Cultural Intermezzo ", New Formations 27 : 128-147.

Bora, Tanil

1996 «Türk Popunda Milliyetcilik

Dalgası», Müzük 1 : 24-25.

Broughton, Simon, et al.

1994 The Rough Guide to World Music.

Londres, Penguin.

Burnett, Robert

1996 The Global Jukebox: The

International Music Industry. Londres,

Routledge.

Cinar, Alev

2001 «Kültürel Bir Kimlik Olarak

Delikanlılıgın Yukselisi'», Dogu-Bati 4 (15) : 141-151.

Du Gay, Paul \& Stuart Hall, eds

1996 Questions of Cultural Identity.

Londres, Sage.
ErImann, Veit

1996 «Aesthetics of the Global

Imagination : Reflections on World Music in the 1990s", Public Culture 8(3) : 467-88.

1999 Music, Modernity and the Global Imagination: South Africa and the West.

Oxford, Oxford University Press.

\section{Feld, Steven}

1994 "From Schizophonia to

Schismogenesis : On the Discourses and Practices of World Music and World Beat ", in Charles Keil \& Steven Feld., eds, Music Grooves. Chicago, Chicago University Press : 257-89.

2000 "The Poetics and Politics of Pygmy Pop " in Georgina Born \& David Hesmondhalgh, eds, Western Music and Its Others: Difference, Representation and Appropriation in Music. Berkeley, University of California Press : 254-279.

Frith, Simon

2000 "The Discourse of World Music ", in Georgina Born and David Hesmondhalgh, eds, Western Music and Its Others:

Difference, Representation and Appropriation in Music. Berkeley, University of California Press : 305-322.

\section{Gilroy, Paul}

1993 The Black Atlantic: Modernity and Double Consciousness. Cambridge, Harvard University Press (trad. franç. : Paris, Kargo, 2003).

Gross, Joan et al., eds

1997 "Arab Noise and Ramadan Nights :

Rai, Rap and Franco-Maghrebi Identity", Diaspora 3 (1) : 3-39.

Guilbault, Jocelyne

1993 Zouk: World Music in the West Indies. Chicago, Chicago University Press. 
1997 «Interpreting World Music: A

Challenge in Theory and Practice ", Popular

Harker, Dave

1997 «The Wonderful World of IFPI : Music Industry Rhetoric, The Critics and The Classical Marxist Critique ", Popular Music 16 (1) : 45-80.

\section{Hesmondhalgh, David}

2000 «International Times: Fusions, Exoticism and Anti-racism in Electronic Dance Music » in Georgina Born \& David Hesmondhalgh, eds, Western Music and Its Others: Difference, Representation and Appropriation in Music. Berkeley, University of California Press : 280-304.

\section{Kastoryano, Riva}

1995 "Etre Turc en France revisité ", Les Annales de l'Autre Islam 3: Turcs d'Europe... et d'ailleurs. Paris, ERISM : 83-92.

\section{Kozanoglu, Can}

1995 Pop Cagi Atesi. Istanbul, Iletisim Yayinevi.

\section{Laing, Dave}

1986 "The Music Industry and "The Cultural Imperialism" Thesis ", Media, Culture and Society $8: 331-41$.

\section{Lipsitz, George}

1994 Dangerous Crossroads: Popular Music, Postmodernism and the Poetics of Place. London, Verso.

\section{Meintjes, Louise}

1990 «Paul Simon's Graceland, South Africa, and the Mediation of Musical Meaning ", Ethnomusicology 34 (1) : 37-73.

Mitchell, Tony

1996 Popular Music and Local Identity. Londres. Leicester NH, Wesleyan University Press.

\section{Negus, Keith}

1997 Popular Music in Theory:

An Introduction. Hanover,University Press of New England.

\section{Regev, Motti}

1997 «Rock Aesthetics and Musics of the World", Theory, Culture and Society 14 (3) : 125-42.

\section{Robins, Kevin \& Morley, David}

1996 "Almanci, Yabanci ", Cultural Studies 10 (2) : 248-254.

\section{Sassen, Saskia}

1998 Globalization and Its Discontents: Essays on the new Mobility of People and Money. New York, The New Press.

Sharma, Sanjay, et al., eds

1996 Dis-orienting Rhythms: The Politics of the New Asian dance music. London, Zed Books.

\section{Slobin, Mark}

1993 Subcultural Sounds: Micromusics of the West. Hanover, University Press of New England.

\section{Spivak, Gayatri}

1988 "Can the Subaltern Speak? " in Nelson Cary \& Lawrence Grossberg, eds, Marxism and the Interpretation of Cultures. Chicago, University of Illinois Press : 271313.

\section{Stock, Johnathan}

1993 "The Application of Schenkerian Analysis to Ethnomusicology: Problems and Possibilities ", Music Analysis 12 (2): 215-40.

\section{Stokes, Martin}

2002 "Turkish Rock and Pop", Garland Encyclopedia of World Music VI: The Middle East. Routledge : 247-253. 
1999 "Sounding Out : The Culture Industries and the Globalisation of Istanbul ", in Caglar Keyder, ed., Istanbul: Between Global and Local. New-York

Rowman and Littlefield, Lanham : 121-139.

Stokes, Martin, ed.

1997 Ethnicity, Identity and Music:

The Musical Construction of Place. Oxford, Berg (Introduction : 1-40).

\section{Taylor, Timothy}

1997 Global pop: World music, World markets. Londres, Routledge.

\section{Tenzer, Michael}

2000 Gamelan Gong Kebyar: The Art of Twentieth Century Balinese Music. Chicago, Chicago University Press.
Tomlinson, John

1991 Cultural Imperialism. Londres,

Pinter.

Virolle, Marie

1995 La Chanson Raï: De l'Algérie profonde à la scène internationale. Paris, Karthala.

Wallis, Roger \& Malm, Krister

1984 Big Sounds from Small Peoples.

London, Constable.

Yalçin-Heckmann, Lale

2002 «Negotiating Identities: Media Representations of Different Generations of Turkish Migrants in German ", in Deniz Kandiyoti, ed., Fragments of Identity: The Everyday of Modern Turkey. Londres, Tauris : 308-321 
Martin Stokes, Musique, identité et "villemonde ". - La mondialisation a vu la formation moderne de l'« identité " se complexifier et les débats à la fois politiques et académiques à propos de sa catégorisation s'intensifier. La position centrale de ce concept a récemment fait l'objet de critiques, en anthropologie autant qu'en musicologie: beaucoup suggèrent, de manière convaincante, que nous malmenons notre compréhension de l'égo contemporain et des processus culturels en insistant sur le fait qu'ils se résument à la " construction de l'identité " et à la production de différence. Cette article émet l'idée que la construction de l'identité et la "production de différence » doivent effectivement être considérées comme des processus culturels centraux de la vie urbaine moderne, mais cherche des moyens d'intégrer ces processus à la vie quotidienne des communautés, des corporations et des villes. Cette approche fait ressortir les identités culturelles comme des processus contestés, en constante construction et dissolution. L'activité musicale, dont les significations sont souvent particulièrement difficiles à établir, fournit un point de référence très utile. L'étude de cas entreprise dans cette article concerne Cartel, un groupe de hip-hop germano-turc, dont les propos identitaires très contestés nécessitent une interprétation mêlant plusieurs contextes - la migration dans l'Europe d'après 1989, la world music dans l'industrie musicale, l'islamisme turc et la transformation d'Istanbul en ville-monde.
Martin Stokes, Ethnicity, Identity and The Global City. - Globalization has complicated modern ways of configuring identity, and intensified both political and scholarly struggles over the category of "identity". The centrality of the term has recently been subject to criticism, both in anthropology and musicology, where many argue, persuasively, that we do a violence to our understanding of contemporary selves and cultural processes by insisting that they are only about «identity construction" and the production of difference. This article suggests that identity construction and "difference producing" still need to be understood as central cultural processes in modern urban life, but seeks ways of embedding these processes in the everyday life of communities, corporations and cities. Cultural identities, in this view of things, will appear as contested processes constantly under construction and dissolution. Musical activity, whose meanings are often particularly hard to fix, provides a useful vantage point. The case study in this article concerns Cartel, a GermanTurkish hip-hop group, whose self-conscious and highly contested identity politics need to be understood in the intersecting contexts of migrancy in Europe post 1989, world music in the recording industries, Turkish Islamism, and "global» refashioning of Istanbul. 